\title{
Food security
}

\author{
Peter Brandt
}

Eingegangen: 2 August 2010/Online publiziert: 18 August 2010 (C) Springer Basel AG 2010

Sicher nicht nur, weil die US-Präsidenten-Gattin Michelle Obama sich und ihre Familie erklärtermaßen gesund ernähren möchte, hat sie zusammen mit 26 Schulkindern von der Bancroft Elementary School in Washington im Frühjahr 2009 im Süden des Gartens des Weißen Hauses einen Nutzgarten von rund 100 Quadratmetern für Obst, Gemüse und Kräuter angelegt. ${ }^{1}$ Sie wollte mit diesem Nutzgarten zugleich ein Zeichen für gesunde Ernährung setzen - unter anderem auch im Hinblick auf die amerikanischen Kinder, von denen viele übergewichtig sind.

Darüber hinaus steht Michelle Obama mit ihrem Anlegen eines Gemüsegartens in Washington in der Tradition der Bewegung "Ackern in der Stadt“, die bereits in den siebziger Jahren mit dem Einrichten von sog. "Community Gardens“ in New York begann. Waren diese „Community Gardens“ damals noch ein Mittel, um den sozialen Zusammenhalt in verfallenden Stadtvierteln $\mathrm{zu}$ befördern, so hat sich inzwischen die auf regionale und saisonale Lebensmittel setzende „Slow-Food-Bewegung“ dieser produktiven „grünen Lungen“ in den Großstädten angenommen. Wenn auch dieses „Ackern in der Stadt“ momentan mit wohl etwas zuviel Enthusiasmus bereits als Ende der modernen Agrarwirtschaft proklamiert wird (Pollan 2006), so hat doch John Hantz - durch Investition von \$ 30 Mill. - in der ehemaligen Autometropole Detroit (Schrumpfen der Einwohnerzahl von knapp zwei Millionen auf weniger als 900.000 Einwohner) das weltweit größte Urban-Farming-Projekt etabliert.

Prof. Dr. Dr. P. Brandt ( $\square)$

Eltviller Str. 5a, 13465 Berlin, Germany

e-mail: Brandt.Orplid@googlemail.com
Inzwischen ist das Urban-Farming (oder UrbanAgriculture) eine (fast) weltweite Bewegung, die sich dem Anbau, der Aufarbeitung und dem Vertrieb von Lebensmitteln in einer Stadt oder ihrem Umfeld verschrieben hat; dieses Urban-Farming erstreckt sich mittlerweile auch auf Tierzucht, Aquakultur, Forstwirtschaft und Gartenbau (Bailkey und Nasr 2000; Hampwaye et al. 2000). Dabei wird - wohl auch leicht weltanschaulich motiviert - in Anspruch genommen, dass das Urban-Farming sowohl zur „Food Security“ als auch zur „Food Safety“ maßgeblich beitragen und außerdem dabei diversen Umweltbelangen gerecht werden soll (Lineberger und Zajicek 2000).

Exemplarisch soll für Deutschland hier in diesem Zusammenhang auf die Gründung der Firma „Nomadisch grün“ von Clausen und Shaw in Berlin hingewiesen werden, deren Kreuzberger Gemeinschaftsgarten uneingeschränkt mobil ist. Durch z. B. den Anbau von Salat, Radieschen und Fenchel in ausgemusterten Brotkisten oder dem Keimen von Setzlingen in Mini-Treibhäusern aus Plastikflaschen sind dem Wechsel auf andere Standflächen innerhalb der Stadt prinzipiell keine Grenzen gesetzt.

Es kann nicht verwundern, dass - in Anbetracht der zukünftigen Entwicklung der Weltbevölkerung und den Folgen des Klimawandels (Lotze-Campen und Schellnhuber 2009; Swiaczny und Schulz 2009; von Koerber et al. 2009; Schneider 2009; Schug 2009; Müller 2009; Stehr und von Storch 2009) - diese Erwägungen, innerhalb von Großstädten Flächen zu schaffen, um raumnah Lebensmittel zu produzieren, schon seit einigen Jahren ernstlich diskutiert werden.

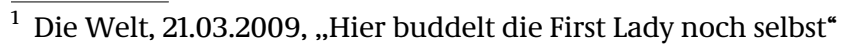


Insofern kann es auch nicht verwundern, dass die bisherigen Anstrengungen des „Urban Farming“ den zukünftigen Anforderungen der „Food Security“ so erscheinen, als ob sie ihnen keineswegs gerecht werden könnten.

Aufgrund der augenblicklichen Situation, dass 6,8 Milliarden Menschen auf der Erde für Ackerbau und Viehzucht bereits eine Fläche benutzen, die der Größe von Südamerika entspricht, und aufgrund der prognostizierten Zunahme der Weltbevölkerung auf 9,5 Milliarden im Jahr 2050 und dem damit verbundenen Mehrbedarf an Ackerfläche in der Größe von Brasilien kommt Despommier (2010a, b) zu dem Schluss, dass soviel „neue“ Ackerfläche einfach nicht verfügbar sein wird. Deshalb schlägt Despommier die Entwicklung und den Bau von Gewächshochhäusern in den Großstädten vor. Seiner Ansicht nach würden diese Gewächshochhäuser über das ganze Jahr Nahrung liefern, dabei weniger Wasser verbrauchen, kaum Abfall produzieren, Infektionskrankheiten eindämmen und keine fossilen Treibstoffe für Landmaschinen oder für den Transport von entlegenen ländlichen Bauernhöfen benötigen. Es ist hier nicht der Ort, um die Vision von Despommier in allen Einzelheiten diskutieren zu können; aber in Anbetracht, dass z. B. die Gestehungskosten (Bodenpreise in Großstädten) und Betriebskosten solcher Gewächshochhäuser derzeit nicht mit letzter Sicherheit abzuschätzen sind, besteht vor der Umsetzung dieser Vision noch viel Klärungsbedarf. Ungeachtet dessen werden Städte als grundsätzlich nachhaltiger angesehen (Pollan 2006); damit wird auch anerkannt, dass das Potential des „Urban Farming“ viel besser genutzt werden muss (Pollan 2006).

Einen anderen, nicht weniger visionären Weg beschreiten diejenigen, die mit Hilfe in sich geschlossener (autarker) Gewächshausanlagen installiert in ariden Landstrichen die dort meist sehr intensive und stets verfügbare Sonneneinstrahlung nutzen wollen, um im großen Stil Nutzpflanzen zu kultivieren und ganzjährig ernten zu können. Die Vorteile einer solchen Vorgehensweise, wie sie diese darlegen, sind fast identisch mit denen von Despommier für seine Gewächshochhäuser.

Angesichts von 875 Mill. unterernährten Menschen weltweit im Jahr 1970 und dem Anstieg auf 1.100 Mill. unterernährter Menschen weltweit im Jahr 2009 (Abb. 1) - trotz aller weltweiten Deklarationen und Aktivitäten in diesen 39 Jahren - stellt sich unweigerlich die Frage, ob solche visionären Projekte von Gemüsehochhäusern in Großstädten oder von autarken Großraum-Gewächshausanlagen in Wüstengebieten derzeit Mittel der Wahl für die
Problemlösung der zukünftigen Welternährung sein können. Doch eine Rückbesinnung auf die derzeitigen Gegebenheiten stellt sich nicht weniger problematisch dar: Wer wollte ernsthaft „völlig selbstlos“ behaupten, in wiefern der Anbau von Pflanzen in konventioneller Weise oder auf der Basis bio-ökologischer Gesichtspunkte oder von transgenen Pflanzen den Anforderungen der zukünftig anwachsenden Weltbevölkerung gerecht werden kann.

Zugestandener Maßen ist dieser zuletzt genannte Erwägungsgrund gravierend belastet von geradezu weltanschaulichen Divergenzen; dennoch sollte dies kein Grund dafür sein, über das Für und Wider dieser verschiedenen Anbauverfahren von Kulturpflanzen angesichts der zukünftigen weltweiten Bedrohung der „Food Security“ intensiv zu diskutieren. Ohne Zweifel sollten dabei auch die in Bezug auf nachhaltige Aspekte relevanten Eigenschaften von konventionell gezüchteten und transformierten Kulturpflanzen nicht außer Acht gelassen werden.

Zwei weitere Aspekte sollten in diesem Zusammenhang nicht unerwähnt bleiben; dies sind die freie Verfügbarkeit von Wasser für alle und die Erzeugung von Treibstoff aus Kulturpflanzen. Aus heutiger Sicht ist die freie Verfügbarkeit von Wasser für alle in keiner Weise sichergestellt und birgt insofern ein unkalkulierbares Risiko lokaler und internationaler Auseinandersetzungen (BrabeckLetmathe 2009); ohne eindeutige internationale Regelung zur Verfügbarkeit von Wasser erscheint jegliche weitere Diskussion über die Wahl des Anbauverfahrens von Kulturpflanzen als obsolet. Nicht minder zweifelhaft erscheinen - angesichts der zu erwartenden Zunahme der Weltbevölkerung und ihres Nahrungsmittelbedarfs - die immer mehr um

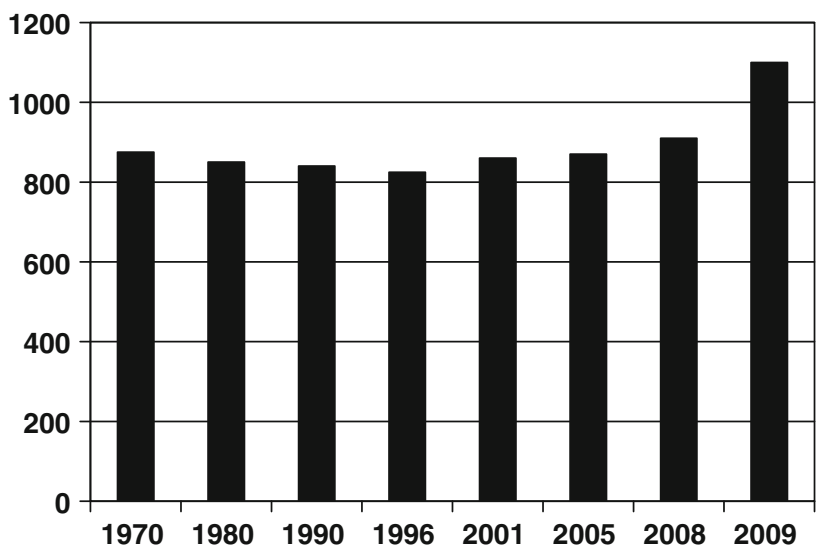

Abb. 1 Anzahl unterernährter Menschen weltweit von 1970 bis 2009 (in Millionen) (Quelle: FAO) 
sich greifenden Aktivitäten zur Herstellung von Treibstoff aus Kulturpflanzen, die eigentlich für die Lebensmittelproduktion vorgesehen sind (Abdulai und Ramcke 2009; Reijnders 2009). In diesem Zusammenhang sollte aber auch erwähnt werden, dass für Pollan (2006) bereits der übermäßige Anbau von Mais und die Gewinnung von Glucose-FructoseSirup aus ihm für etliche Lebensmittelinhaltstoffe vom Grundprinzip der nachhaltigen Lebensmittelversorgung weggeführt hat. Ohne Zweifel kann es wohl keine stichhaltigen Gründe geben, die Gewinnung von Biotreibstoff aus Kulturpflanzen zu propagieren, die eigentlich der Erzeugung von Lebensmitteln für den menschlichen Verzehr zugedacht sind.

Wenn überhaupt, so kann die ausreichende Ernährung der Weltbevölkerung in Zukunft nur dann sicher gestellt werden („Food Security“), wenn - jenseits des momentanen Profits - (a) die Produktion pflanzlicher Lebensmittel auf der Basis der verfügbaren Methoden optimiert wird, (b) die globalen Probleme der Verteilung von Lebensmitteln gelöst werden und (c) auf die Ernährungsgewohnheiten der Menschen in nachhaltiger Weise erfolgreich eingewirkt werden kann.

\section{Literatur}

Abdulai A, Ramcke L (2009) Acute View: Auswirkungen der Biokraftstoffproduktion auf die Ernährungssicherheit. J Verbr Lebensm 4:160-163

Bailkey M, Nasr J (2000) From brownfields to greenfields: producing food in North American cities. Community Food Security News. Fall 1999/Winter 6
Brabeck-Letmathe P (2009) Energy and water. J Verbr Lebensm 4:155-159

Despommier D (2010a) Das Gewächshaus im Wolkenkratzer. Spektrum der Wissenschaft April 2010, pp 72-80

Despommier D (2010b) Vertical farm: the big idea that could solve the world's food, water and energy crises. Thomas Dunne Books/St. Martin's Press, New York

Hampwaye G, Nel E, Ingombe L (2000) The role of urban agriculture in addressing household poverty and food security: the case of Zambia. GDN Working Paper

Lineberger SE, Zajicek JM (2000) Can a hands-on teaching tool affect students' attitudes and behavior regarding fruit and vegetables? Hort Technol 10:593-596

Lotze-Campen H, Schellnhuber H-J (2009) Climate impacts and adaptation options in agriculture: what we know and what we don't know. J Verbr Lebensm 4:145-150

Müller G (2009) Die Welternährung im Jahr 2030 unter besonderer Berücksichtigung der Bevökerungsentwicklung, der sich ändernden Flächenpotenziale und des Klimawandels. J Verbr Lebensm 4:52-55

Pollan M (2006) The omnivore's dilemma: a natural history of four meals. Penguin Press, Harmondsworth, pp 450

Reijnders L (2009) Acute view: transport biofuels-can they help limiting climate change without an upward impact on food prices? J Verbr Lebensm 4:75-78

Schneider R (2009) Welternährung sichern im Klimawandel. J Verbr Lebensm 4:39-43

Schug W (2009) Die Entwicklung der Weltbevölkerung und die globale Nahrungsmittelversorgung. J Verbr Lebensm 4:4451

Stehr N, von Storch H (2009) Climate Protection. J Verbr Lebensm 4:56-60

Swiaczny F, Schulz R (2009) Wachstum der Weltbevölkerung und nachhaltige Tragfähigkeit. J Verbr Lebensm 4:136-144

von Koerber K, Kretschmer J, Prinz S, Dasch E (2009) Globale Nahrungssicherung für eine wachsende Weltbevölkerung-Flächenbedarf und Klimarelevanz sich wandelnder Ernährungsgewohnheiten. J Verbr Lebensm 4:174-189 